\title{
4/3-Law of Granular Particles Flowing through a Vertical Pipe
}

\author{
Osamu Moriyama, Naoya Kuroiwa and Mitsugu Matsushita \\ Department of Physics, Chuo University, Kasuga, Bunkyo-ku, Tokyo 112-8551, Japan \\ Hisao Hayakawa \\ Graduate School of Human and Environmental Studies, Kyoto University, Sakyo-ku, Kyoto 606-8501, Japan
}

(Received June 16, 1997)

Density waves of granular material (sand) flowing through a vertical pipe have been investigated. Clear density waves emerge when the cock attached to bottom end of the pipe is closed. The FFT power spectra were found to show a stable power-law form $P(f) \sim f^{-\alpha}$. The value of the exponent was evaluated as $\alpha \cong 4 / 3$. We also introduce a simple onedimensional model which reproduces $\alpha=4 / 3$ from both simulation and theoretical analysis.

Recently much attention has been paid to the dynamics and statistics of granular materials because of their ubiquity in nature and the application to technology. Unlike usual solids, liquids or gases, granular materials are known to show complex dynamical behaviors [1], such as convection [2], size segregation [3], bubbling [4], standing waves and localized excitations under vertical vibration [5.6] and a fluidized bed due to air injected inside a box containing granules [7],8].

Pattern formation of grains flowing through a vertical pipe which can be regarded as a one dimensional realization of a fluidized bed is also a typical example of unusual features of granular motion $9 \sqrt{13}$. Emergence of density waves (e.g. slugging) has been investigated by molecular dynamics (MD) and lattice-gas automata (LGA) simulations [9, 10] and by the experiments using sand in air 111,12 and metallic spheres in liquids 13. Power-law form of the power spectrum $P(f) \sim f^{-\alpha}$, where $f$ is frequency, of density fluctuations was also found in both numerical simulations [9] and experiments [11,12]. Although their interpretations on the origin of the emergence of density waves are different, estimated values of the exponent $\alpha$ is close to each other $(1.3<\alpha<1.5)$.

Although the previous experiment 11.12] reported $\alpha \cong$ 1.5, some of their experimental procedures seem a little ambiguous: Since they merely plugged up the bottom hole of a pipe by half in order to induce density waves, the rate of air flow out of the bottom end of the pipe was not well controlled. Besides, the power spectra they obtained were still noisy. In this Letter we will first present bettercontrolled air flow out of the pipe and more accurate experimental results than the previous ones by increasing the number of trials. One of our results is the precise estimation of the scaling exponent of the power spectrum
$P(f) \sim f^{-\alpha}$. The result is $\alpha \cong 4 / 3$. We also propose a one-dimensional model supplemented by the white noise which reproduces $\alpha=4 / 3$ near the neutral curve of the linear stability analysis of uniform states. In other words, we will clarify the origin of the power-law in density waves of granular pipe flows.

FIG. 1. Schematic illustration of the experimental setup.

Our experimental setup is shown in Fig. 1. We used a glass pipe of $1500 \mathrm{~mm}$ length and $3 \mathrm{~mm}$ inner diameter, i.e., the same one used in Ref. [11, 12]. A flask is connected to the bottom end of the vertical pipe and a flow-meter is attached to an outlet stuck out of the flask. We used rough sand, i.e., our granular particles are polydisperse and their average diameter is about 0.3 $\mathrm{mm}$. Thus the inner diameter of the pipe is about ten times larger than the average diameter of sand. We pored rough sand into a hopper connected to the top end of the pipe, which flows through the pipe due to gravity. Sand finally falls into the flask while air can exit out of it through the flow-meter, which can control the rate of air discharge. Meanwhile we measured density fluctuations as the transmission light intensity across the pipe at a fixed location midway up the pipe by using laser light and detecting system (KEYENCE, LX2-02). The laser light has a rectangular cross-section of $10 \mathrm{~mm}$ wide and $1 \mathrm{~mm}$ high and is emitted with a pulse frequency of $4096 \mathrm{~Hz}$. The detecting system has linear response between the output voltage and the density of particles in the range of output voltage between 1 and 5 volts.

Let us now consider what happens by closing the cock of flow-meter. Granular particles falling in a pipe inter- 
act with the medium (air) due to the viscosity. When the flow-meter is removed from the apparatus, i.e., the bottom end of the pipe is fully open, granules can fall rather freely as if the existence of air could be neglected. (Hereafter we will refer this situation to fully open.) In fully open there are no visible density waves, as reported in Refs. 11, 12]. The reason is that both granules and air flow together through the pipe. As the cock is gradually closed, however, the pressure in the flask rises and the effect of the viscous force becomes more important. In particular, when the cock is fully closed (we refer to fully closed), air in the pipe must go upward due to approximate conservation of the total volume (sand plus air) in the flask while sand falls downward. We consider that the increment of interaction between granules and air induces density waves.

FIG. 2. Time series signal of the granular flows for fully open (top) and fully closed (bottom). Upper signal is shifted by $0.4 \mathrm{~V}$ to avoid the data overlap

Figure 2 shows typical time series signals of granular flows measured at $x=100 \mathrm{~cm}$, where $x$ is defined as the distance of measuring point along the pipe from the top. In contrast with the white-noise-like signal (top) in fully open, one can see that the density wave for fully closed (bottom) has intermittent structure. In this figure higher (lower) voltage corresponds to smaller (larger) granular density since we measured the light intensity transmitted across the pipe. In particular, the output voltage is about 2.15 volts when a pipe is fully packed with sand, and about 2.50 volts when it is completely empty. This assures us that our measurements were well in the linear range between the voltage and the density. In the case of real flow patterns one can observe that two clusters sometimes collide with each other and merge into one, and sometimes one cluster splits into more than one cluster, in a behavior reminiscent of a chain of traffic jams on a crowded highway 14 16. In the following discussion of this Letter we restrict ourselves to fully closed. (When the medium air was controlled to flow downward by gradually opening the cock of the flow-meter, the position of the emergence of clear density waves gradually shifted downward. The details of the results will be presented elsewhere.)

FIG. 3. Log-log plot of power spectra $P(f)$ of time series signals in fully closed. The straight line with the slope of $-4 / 3$ in the figure is for an eye-guide.

Let us pay our attention to the power spectrum $P(f)$ of density fluctuations of granular flows. Figure 3 shows $P(f)$ of recorded signals in fully closed. Each spectrum was obtained by averaging over 640 independent data with length 8192 discrete points each (two seconds in real time) and was appropriately shifted to avoid the overlap. The self-organization of the power-law form $P(f) \sim f^{-\alpha}$ is observed as the measuring position $x$ increases: The spectrum at $x=10 \mathrm{~cm}$ is similar to that in fully open. Density waves have already emerged at $x=40 \mathrm{~cm}$ where the scaling regime is covered by the whole frequency range except for the fast decay in higher $f$. Then the system falls into the steady state from about $x=50 \mathrm{~cm}$ downward. The scaling range for the steady state, i.e., for $x \geq 50 \mathrm{~cm}$ is from $10 \mathrm{~Hz}$ to $200 \mathrm{~Hz}$, as seen in Fig. 3 .

In the frequency range from $15 \mathrm{~Hz}$ to $150 \mathrm{~Hz}$, values of the slope are fitted by the least mean square method as $\alpha=1.33 \pm 0.04,1.31 \pm 0.05$ and $1.34 \pm 0.05$ for $x=80$, 90 and $100 \mathrm{~cm}$, respectively. (The data at $x=90 \mathrm{~cm}$ and $100 \mathrm{~cm}$ are not shown in Fig. 3.) These values are very close to $4 / 3$ suggested by LGA simulation [9].

Let us allocate the rest of this Letter to introduce a one-dimensional model which reproduces $\alpha=4 / 3$ in fully closed system.

The important mechanisms for particle dynamics are 
the drag between air and particles, and the relaxation process to an optimal velocity which may be the sedimentation rate of particles. When the $N$ particles are confined in a quasi-one dimensional container, the motion of particles may be described by the following nonlinear equation:

$$
\ddot{r}_{n}+\zeta\left[\dot{r}_{n}-W\left(\left\{r_{n}\right\}\right)\right]=T F\left(\left\{r_{n}\right\}\right)+f_{n}(t),
$$

where $r_{n}$ and $\zeta$ are the relative distance between the $n$-th and $(n+1)$-th particles, and the drag coefficient, respectively. The collisional force $F\left(\left\{r_{n}\right\}\right)=\varphi^{\prime}\left(r_{n+1}\right)+$ $\varphi^{\prime}\left(r_{n-1}\right)-2 \varphi^{\prime}\left(r_{n}\right)$ comes from a soft core repulsive potential $\varphi\left(r_{n}\right)$. The parameter $T$ represents the strength of repulsion. The optimal velocity $W\left(\left\{r_{n}\right\}\right)=U\left(\frac{r_{n+1}+r_{n}}{2}\right)-$ $U\left(\frac{r_{n}+r_{n-1}}{2}\right)$ is the linear combination of sedimentation rate $U(x)$ which is the nonlinear function of the local volume fraction [17] in general. The most crucial simplification of (11) without any justification is to assume that $f_{n}$ is a Gaussian white noise with zero mean. It should be noticed that the drag $\zeta$ is irrelevant in fully open, because air in the pipe flows away together with particles. Thus, the motion of particles are almost elastic and clear density waves cannot be observed in fully open. It should be noted that (11) is written for the relative motion of particles. Discarding the noise term from (11), we obtain an equation of motion for particle at the position $x_{n}$ as $\ddot{x}_{n}+\zeta\left[\dot{x}_{n}-U\left(\frac{x_{n+1}-x_{n-1}}{2}\right)\right]=T\left[\varphi^{\prime}\left(r_{n}\right)-\varphi^{\prime}\left(r_{n-1}\right)\right]$ where $r_{n}=x_{n+1}-x_{n}$.

Linearizing (11) around the uniform solution $\dot{r}_{n}=0$ where $a=\bar{r}_{n} \equiv N^{-1} \sum_{n} r_{n}$, we obtain

$$
\ddot{\tilde{r}}_{k}+\zeta\left[\dot{\tilde{r}}_{k}-i U^{\prime} \sin k \tilde{r}_{k}\right]=2 T \varphi^{\prime \prime}(\cos k-1) \tilde{r}_{k}+\tilde{f}_{k}(t),
$$

where the argument of $U^{\prime}$ and $\varphi^{\prime \prime}$ is $a . \tilde{r}_{k}$ and $\tilde{f}_{k}(t)$ are respectively the Fourier transform of $\delta r_{n}=r_{n}-a$ and $f_{n}(t)$. Equation (2) has the solution $\tilde{r}_{k}(t) \propto \exp \left[\sigma_{ \pm} t\right]$, where

$\sigma_{ \pm}=-\frac{\zeta}{2} \pm \sqrt{\left(\frac{\zeta}{2}\right)^{2}-2 T \varphi^{\prime \prime}(1-\cos k)+i \zeta U^{\prime} \sin k}$

$\operatorname{Re}\left[\sigma_{+}\right]$represents the relevant eigenvalue of the linear problem, which becomes positive for $U^{\prime}(a)^{2} \cos ^{2}(k / 2) \geq$ $T \varphi^{\prime \prime}(a)$. Thus the most unstable wave number is $k \rightarrow 0$ and the neutral curve is given by $T_{c}=U^{\prime 2} / \varphi^{\prime \prime}(a)$. At $T=T_{c}(1-\mu)$ the expansion of $\sigma_{+}$around $k=0$ is given by $\sigma_{+}(k) \simeq i\left[c_{0} k-\frac{c_{0}}{6} k^{3}+\cdots\right]+\frac{c_{0} \mu}{\zeta} k^{2}-\frac{c_{0}^{2}}{4 \zeta} k^{4}+\cdots$, where $c_{0}=U^{\prime}(a)$. Thus, for $\mu>0$ the uniform state is unstable due to the negative diffusion.

Adopting $U(r)=\tanh (r-2)+\tanh (2), \varphi(r)=\operatorname{sech}^{2}(r)$ $, \zeta=2, N=256, T_{c}=3.95798 \cdots$, and $a=2$ at $t=0$, we simulated (11) by the classical Runge-Kutta method until $t=2^{11}$ with time interval $\Delta t=1 / 2^{4}$ under the periodic boundary condition. We used the uniform random number distributed between $-X$ and $X$ with $X=9 / 1024$ [18] for $f_{n}(t)$. Figure 4 displays the power spectrum
$P(f)=|\tilde{c}(f)|^{2}$ obtained from our simulation of (11) at $\mu=1 / 64$, where $\tilde{c}(f)$ is the Fourier transform of the discretely sampled data of the density $c(t)=\frac{1}{N} \sum_{n} \frac{1}{r_{n}(t)}$ with the interval 1 . This clearly supports $P(f) \sim f^{-4 / 3}$ as in our experiment. From the examinations of several values of $\mu$, we have confirmed that the qualitative results are insensitive to the sign of $\mu$ when $|\mu| \ll 1$. This result is reasonable because near the neutral curve the time scale of relaxation or growth of fluctuations is much longer than the time scale induced by the noise $f_{n}(t)$. Our result suggests that the linear relaxation theory of fluctuations can be used to explain $P(f) \sim f^{-4 / 3}$.

FIG. 4. Log-log plot of power spectrum $P(f)$ obtained from the numerical integration of (1), where the unit of $f$ is $1 /(2 \pi)$. The guide line represents $f^{-4 / 3}$. See the text for details.

Let us briefly sketch how to derive 4/3-law from the behavior of structure factor

$$
S_{k}(t)=\sum_{n, m}<\exp \left[i k\left(\delta r_{n}(t)-\delta r_{m}(0)\right)\right]>
$$

in weakly stable states, i.e., $\mu<0$ and $|\mu| \ll 1$, where $\delta r_{n}=r_{n}-a$ is the fluctuation of relative distance. The structure factor can be rewritten as $S_{k}(t)=$ $\frac{1}{N} \sum_{n, m} \exp \left[-\frac{k^{2}}{2} \phi_{n m}(t)\right]$ where $\phi_{n m}(t)=<\left(\delta r_{n}(t)-\right.$ $\left.\delta r_{m}(0)\right)^{2}>$. For $\mu<0, S_{k}(t)$ can be calculated as in the case of polymer dynamics 19 . With the aid of the expansion of $\sigma_{+}$, (11) is reduced to

$$
\partial_{\tau} r(z, \tau)-\partial_{z}^{3} r(z, \tau)=\epsilon\left[\partial_{z}^{2}-\partial_{z}^{4}\right] r(z, \tau)+\xi(z, \tau)
$$

where $\tau=\epsilon^{3} \beta t, z=\frac{2 \zeta}{3 c_{0}} \epsilon\left(x+c_{0} t\right), \xi(z, \tau)=\epsilon^{3} \beta f_{n}(t)$ with $\epsilon=\frac{3 \sqrt{c_{0}}}{\zeta} \sqrt{-\mu}$ and $\beta=\frac{4}{3 \sqrt{c_{0}}}$. The solution of (5) is given by $\tilde{r}_{k}(\tau) \simeq \int_{0}^{\tau} d s \exp \left[\lambda_{k}(\tau-s)\right] \tilde{\xi}_{k}(s)$, where $\lambda_{k}=i k^{3}-\epsilon k^{2}\left(1+k^{2}\right)$. Thus, we obtain the correlation 


$$
<\tilde{r}_{k}(\tau) \tilde{r}_{-k}(0)>=\frac{D}{2 \epsilon l k^{2}\left(1+k^{2}\right)} \exp \left[\lambda_{k} \tau\right]
$$

where $l$ is the system size, and we used $<\tilde{\xi}_{k}(\tau) \tilde{\xi}_{p}\left(\tau^{\prime}\right)>=$ $\frac{D}{l} \delta_{k+p, 0} \delta\left(\tau-\tau^{\prime}\right)$.

A long but straightforward calculation parallel to Ref. [19] yields

$$
S_{k}(\tau) \simeq \int_{-l}^{l} d x \exp \left[-D_{G} k^{2} \tau-\frac{D k^{2}}{2 \epsilon} w-\frac{D k^{2}}{\pi \epsilon} \tau^{1 / 3} h(u)\right]
$$

where the argument of $S_{k}$ is replaced by the scaled time, $D_{G}$ is the diffusion constant for the gravitational center in (5), $u=x \tau^{-1 / 3}$, and $w=\left|z-z^{\prime}\right|$. Since $h(u)$ converges to $h(0)=\pi / \Gamma(1 / 3)$ as time goes on, we obtain

$$
S_{k}(\tau) \simeq \frac{\epsilon}{D k^{2}} \exp \left[-\frac{D k^{2}}{\epsilon \Gamma(1 / 3)} \tau^{1 / 3}\right]
$$

in intermediate time range. In the limit of small $\tau$, $S_{k}(\tau) \propto 1-\frac{4 D k^{2}}{\epsilon \Gamma(1 / 3)} k^{2} \tau^{1 / 3}+\cdots$. Thus its Fourier transform, giving the power spectrum $P_{k}(f)$ obeys

$$
P_{k}(f) \sim f^{-\alpha}, \quad \alpha=4 / 3 \quad(\text { as } \quad f \rightarrow \infty),
$$

where use was made of $\int_{-\infty}^{\infty} d t e^{i 2 \pi f t}|t|^{1 / 3} \propto f^{-4 / 3}$. The value $4 / 3$ is identical to the one obtained by our experiments and numerical simulations [9]. Thus our model (11) reproduces $\alpha=4 / 3$. It should be noted that the appearance of this power-law form in the original model (11) is only for $f<\zeta$ since we eliminate the fast decaying mode $\sigma_{-}$in our analysis. This tendency is also observed as the higher-frequency cutoff in our experiment (see Fig. 3).

The 4/3-law is determined by short time behavior of the dynamics of density waves induced by the noise, which is essentially determined by the linear dispersion relation $\lambda_{k} \sim i k^{3}$. The details of our theoretical analysis including the effects of nonlinearity will be discussed elsewhere [20].

In this Letter we have confirmed $\alpha=4 / 3$ as the powerlaw exponent in the frequency spectrum of density correlation function from the experiment, the simulation and the theory. We have also clarified the mechanism to yield $f^{-4 / 3}$ spectrum which is related to the critical slowing down of the density fluctuations. It should be noticed that the continuous increase of $\alpha$ in LGA [10] from $\alpha=0$ to 2 with the particle density is consistent with the $4 / 3$ law and our picture, because the spectrum determined by the noise in linearly stable uniform state far from the neutral curve should be white $(\alpha=0)$ and the effective exponent of the power-law becomes large when the exponential decay (i.e. $\alpha=2$ ) in the off-critical region exists. There is, however, a discrepancy between our results and the one on experiments in liquids [13]. The reason of this difference should be clarified in the future.
OM, NK and MM would like to thank Y-h. Taguchi, T. Isoda and A. Nakahara for stimulating discussions. $\mathrm{HH}$ thanks K. Ichiki, K. Nakanishi and G. Peng for fruitful discussions. This work is partially supported by Grantin-Aid for Science Research Fund from the Ministry of Education, Science and Culture (09740314).

[1] H. M. Jaeger, S. R. Nagel and R. P. Behringer, Rev. Mod. Phys. 68, 1259 (1996).

[2] Y-h. Taguchi, Phys. Rev. Lett. 69, 1367 (1992), K. M. Aoki T. Akiyama, Y. Maki and T. Watanabe, Phys. Rev. E 54, 874 (1996).

[3] A. Rosato, K. J. Strandburg, F. Prinz and R. H. Swendsen, Phys. Rev. Lett. 58, 1038 (1987).

[4] H. K. Pak and R. P. Behringer, Nature 371, 231 (1994).

[5] F. Melo, P. B. Umbanhowar and H. L. Swinney, Phys. Rev. Lett. 75, 3838 (1995).

[6] P. B. Umbanhowar, F. Melo and H. L. Swinney, Nature 382, 793 (1996).

[7] G. K. Batchelor, J. Fluid Mech. 193, 75 (1988).

[8] S. Sasa and H. Hayakawa, Europhys. Lett. 17, 685 (1992), T. S. Komatsu and H. Hayakawa, Phys. Lett. A 183, 56 (1993).

[9] G. Peng and H. J. Herrmann, Phys. Rev. E 49, R1796 (1994).

[10] G. Peng and H. J. Herrmann, Phys. Rev. E 51, 1745 (1995).

[11] S. Horikawa, A. Nakahara, T. Nakayama and M. Matsushita, J. Phys. Soc. Japan 641870 (1995).

[12] S. Horikawa, T. Isoda, T. Nakayama, A. Nakahara and M. Matsushita, Physica A 233, 699 (1996).

[13] A. Nakahara and T. Isoda, Phys. Rev. E 55, 4264 (1997).

[14] M. Bando, K. Hasebe, A. Nakayama, A. Shibata and Y. Sugiyama, Phys. Rev. E 51, 1035 (1995).

[15] B. S. Kerner and P. Konhauser, Phys. Rev. E 48, 2335 (1993).

[16] T. S. Komatsu and S. Sasa, Phys. Rev. E 52, 5574 (1995).

[17] H.Hayakawa and K.Ichiki, Phys.Rev. E 51, R3815 (1995) and references therein.

[18] The value of $X$ is chosen to have the unit amplitude in the form of eq.(5).

[19] P.G. de Gennes, Physics 3, 37 (1967): see also M. Doi and S. F. Edwards, The Theory of Polymer Dynamics (Oxford, 1986).

[20] Detailed calculation within the linear theory discussed here can be seen in : H. Hayakawa and K. Nakanishi, Prog.Theor.Phys. Suppl. (to be published). 


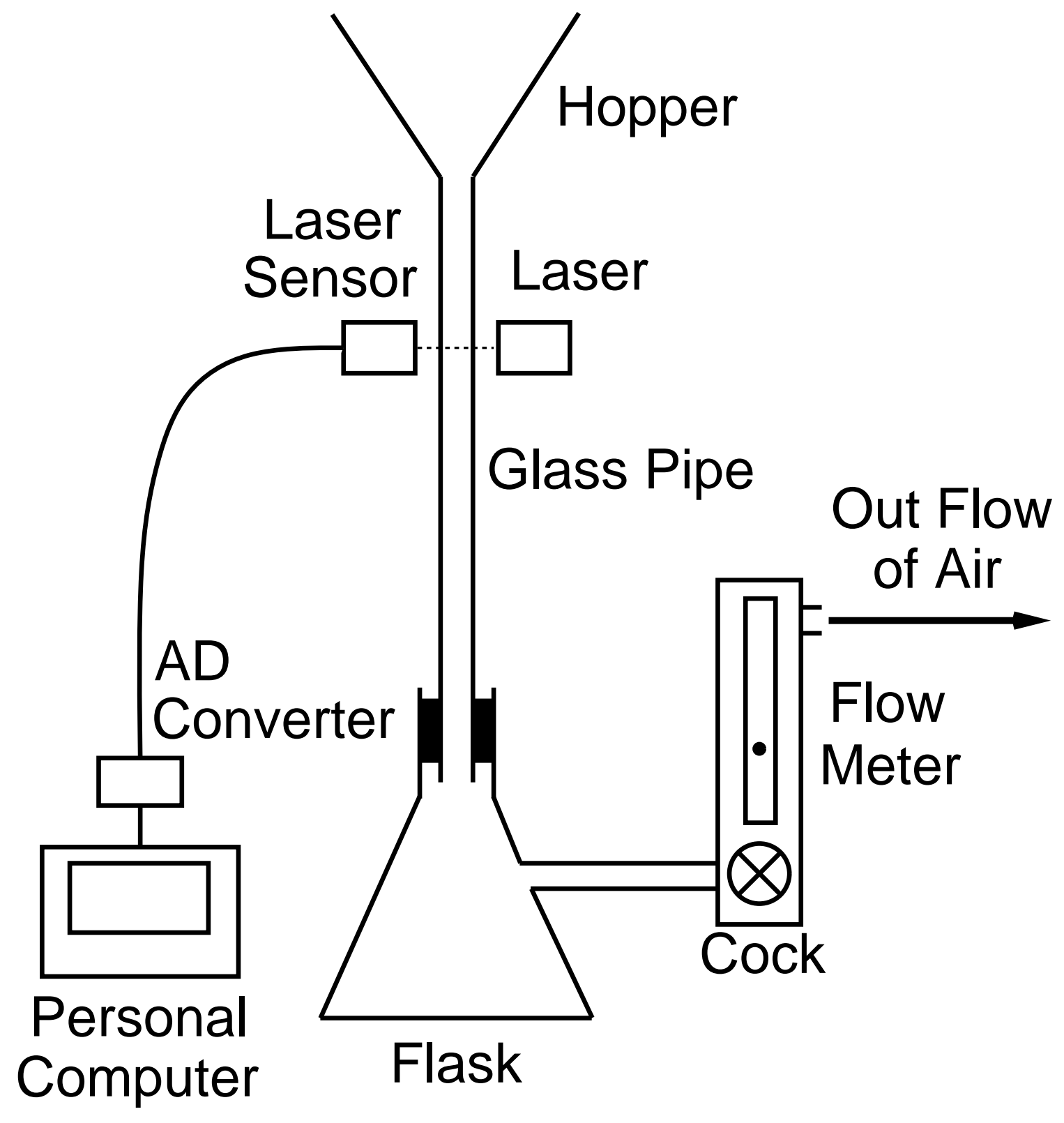




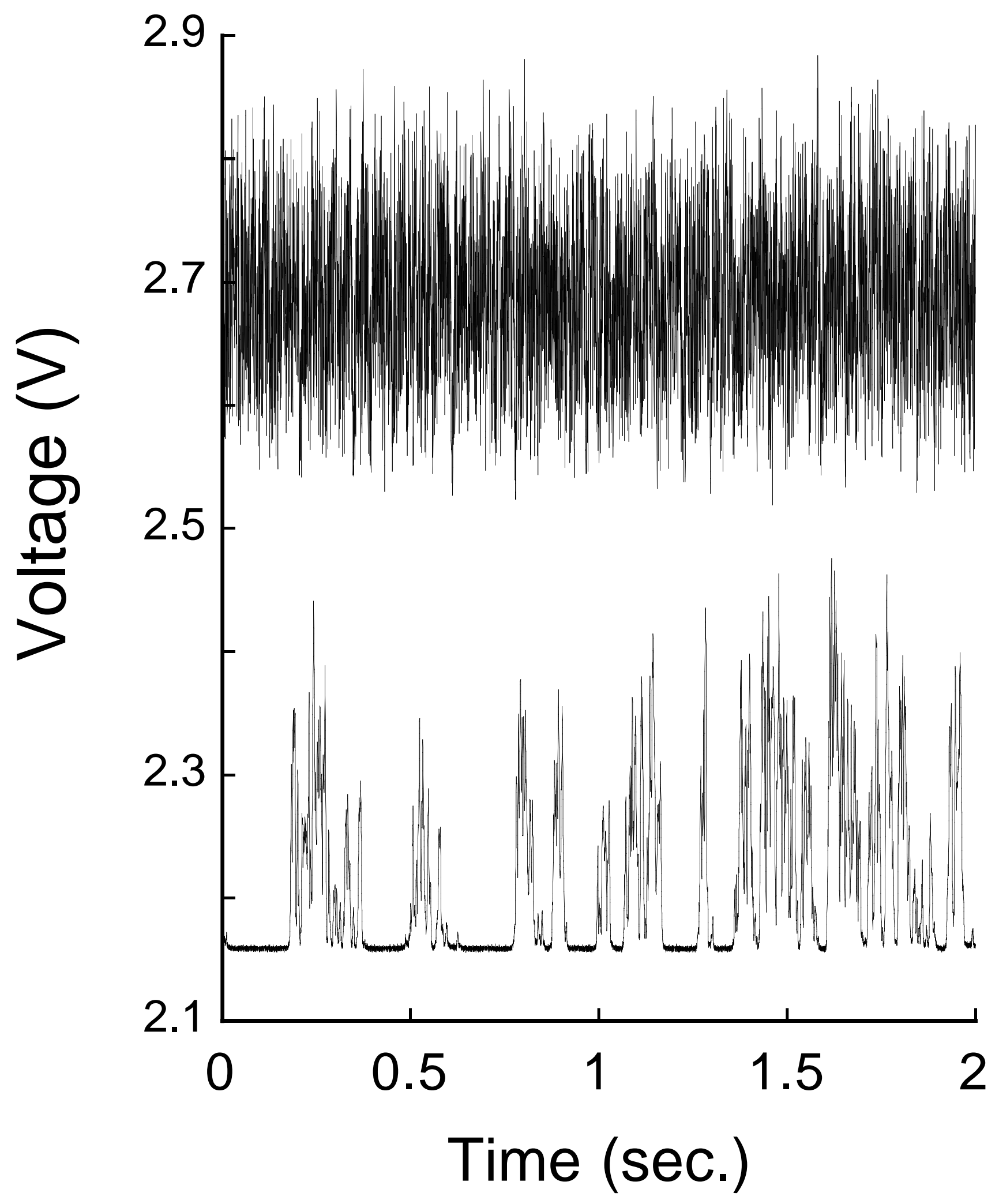




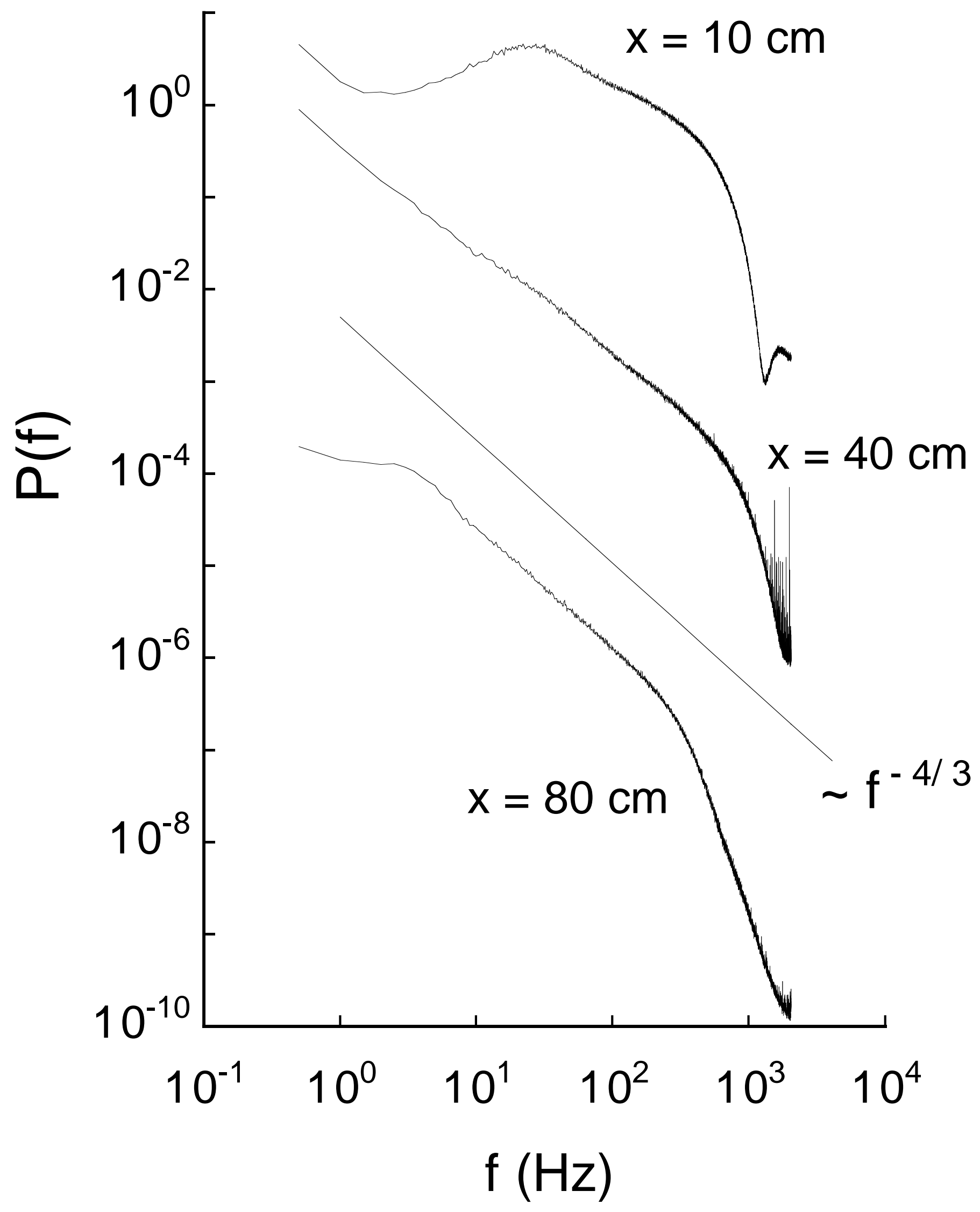




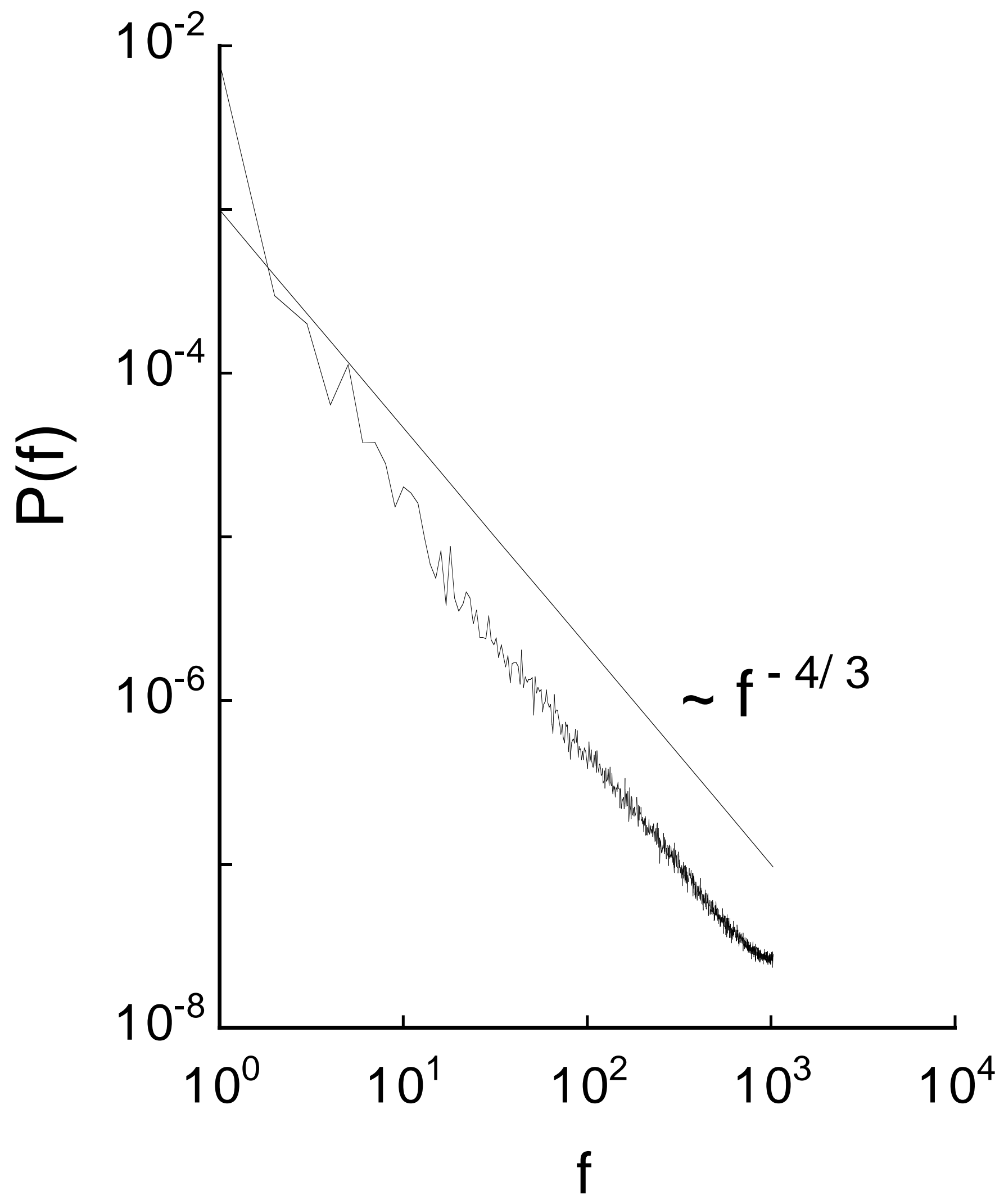

